\title{
A mulher trabalhadora de enfermagem e os distúrbios osteomusculares relacionados ao trabalho
}

FEMALE NURSES AND THE OSTEOMUSCULAR DISTURBANCES RELATED TO THEIR WORK

\author{
LA MUJER TRABAJADORA DE ENFERMERIA Y LOS DISTURBIOS OSTEOMUSCULARES \\ RELACIONADOS AL TRABAJO
}

Patricia Campos Leite ${ }^{1}$, Arlete Silva², Miriam Aparecida Barbosa Merighi ${ }^{3}$

\section{RESUMO}

O presente estudo aborda a relação entre a mulher trabalhadora de enfermagem e os distúrbios osteomusculares relacionados ao trabalho. Evidencia-se que os trabalhadores de enfermagem estão expostos a uma série de condições desfavoráveis no ambiente de trabalho. As queixas predominantes estão relacionadas ao aparelho osteomuscular, que incidem em maior proporção no sexo feminino, em razão não apenas das características biológicas da mulher, mas à desigual divisão sexual do trabalho presente na atualidade.

\section{ABSTRACT}

This study investigates the relation between female nurses and osteomuscular disturbances associated with their work. It is clear from the study that Nursing professionals are exposed to a number of unfavorable conditions in their working environment. Predominant complaints are related to the osteomuscular system, which affects mostly females due not only to the biological characteristics of women, but also because of the uneven distribution of work between genders that prevails nowadays.

\section{RESUMEN}

Este estudio enfoca la relación entre la mujer trabajadora de enfermería y los disturbios osteomusculares relacionados al trabajo. Es evidente que los profesionales de enfermería están expuestos a una serie de condiciones desfavorables en el ambiente de trabajo, siendo predominantes las quejas relacionadas al aparato osteomuscular, que inciden en mayor proporción en el sexo femenino, en razón no apenas de las características biológicas de la mujer, más también a la desigual división sexual del trabajo existente en la actualidad.

\author{
1 Doutora em \\ enfermagem pela \\ EEUSP. Professora \\ da disciplina de \\ Enfermagem em \\ Centro Cirúrgico do \\ Centro Universitário \\ Nove de Julho. \\ patipavan@uninove.br \\ 2 Professora Doutora \\ pela EEUSP. \\ Professora da \\ Universidade de \\ Guarulhos-UNG. \\ 3 Professora Livre- \\ Docente do \\ Departamento de \\ Enfermagem \\ Materno-Infantil e \\ Psiquiatria da \\ EEUSP. Líder do \\ Grupo de Pesquisa \\ Enfermagem e a \\ Subjetividade da \\ Mulher no Processo \\ Saúde-Doença. \\ merighi@usp.br
}

\section{DESCRITORES}

Transtornos traumáticos cumulativos.

Saúde da mulher.

Enfermagem do trabalho.

\section{KEY WORDS}

Cumulative trauma disorders. Women's health. Occupational health nursing.

\section{DESCRIPTORES}

Transtornos de traumas acumulados.

Salud de la mujer.

Enfermería del trabajo. 


\section{INTRODUÇÃO}

Vivemos um momento histórico inteiramente invadido pelo capitalismo, no qual as relações de consumo são predominantes no modo de ser e agir dos indivíduos, as inovações tecnológicas e as intensas transformações do mundo globalizado tendem a acarretar profundas mudanças no modo de viver das pessoas, inclusive no âmbito profissional.

Nota-se que muitos dos avanços conquistados pela humanidade, além dos benefícios, ocasionam, problemas à saúde dos trabalhadores ${ }^{(1)}$.

Nessa perspectiva, a literatura científica demonstra que as condições de trabalho vivenciadas pelos profissionais da equipe de enfermagem em vários países da América do Sul, são consideradas piores àquelas vividas pelos enfermeiros americanos e europeus. Esse fato justifica-se pelas sérias dificuldades políticas e econômicas enfrentadas pelos países em desenvolvimento. Além disso, o desgaste físico e emocional, a baixa remuneração e o desprestígio social são fatores associados às condições de trabalho do enfermeiro, que vem refletindo negativamente na qualidade da assistência prestada ao cliente, levando ao abandono da profissão e conseqüentemente a escassez de profissionais no mercado de trabalho ${ }^{(2)}$.

Os trabalhadores de enfermagem estão expostos a uma série de situações de risco durante a execução de seu trabalho, que podem ocasionar acidentes e doenças ocupacionais ${ }^{(3)}$.

Os acidentes do trabalho, de uma maneira geral, são mais facilmente notificados comparados às doenças ocupacionais, as quais requerem uma avaliação e comprovação do nexo causal para serem reconhecidas como tal, ocasionando a subnotificação dos dados de adoecimento dos trabalhadores.

Dessa forma, atualmente existe escassez de informações sobre o perfil de adoecimento dos trabalhadores de enfermagem, destacando a importância de investigações sobre o perfil de morbidade dessa categoria ${ }^{(4)}$.

Considerando que a equipe de enfermagem é composta predominantemente pelas mulheres, é importante que se reconheçam as diferenças inerentes ao gênero para qualquer análise do processo saúde-doença dessa classe trabalhadora. Assim, enquanto as atividades domésticas, geralmente atribuídas às mulheres, não são consideradas "trabalho" pois tratar-se-iam apenas de atividades de manutenção das condições para a realização do autêntico trabalho, a desigualdade entre os sexos é perpetuada, causando maiores agravos à mulher trabalhadora ${ }^{(5)}$.

Entre os principais problemas de saúde que acometem a mulher trabalhadora de enfermagem, destacam-se os relacionados ao aparelho ostomuscular, os quais têm sido elucidados por vários trabalhos ${ }^{(6-7)}$.
Nesse sentido, o presente artigo trata-se de um estudo teórico que tem como objetivo descrever a relação entre a mulher trabalhadora de enfermagem e a ocorrência dos distúrbios osteomusculares relacionados ao trabalho (DORT).

\section{DISTÚRBIOS OSTEOMUSCULARES RELACIONADOS AO TRABALHO}

As relações de trabalho evidenciadas pela grande competitividade, pelos elevados níveis de exigência e produtividade, são fatores que consequientemente promovem alterações no processo saúde-doença de toda a humanidade.

A exposição dos trabalhadores às inúmeras modalidades e intensidades de riscos, intermediados pelas particularidades dos processos industriais ocorreram à medida em que houve intensa utilização de diferentes recursos tecnológicos associado às diversificadas formas de controle e organização, desencadeando não somente insatisfação e apatia pelo trabalho, como elevação nos perfis de morbidade da classe trabalhadora ${ }^{(8-9)}$.

Em meio a tantos progressos é possível perceber que o adoecimento e a morte como conseqüência do trabalho faz parte da história do homem e algumas das doenças do trabalho são quase tão antigas quanto ele, havendo uma relação estreita com o processo de trabalho e muitas das doenças e acidentes ocorridos com os trabalhadores ${ }^{(10)}$.

Importante citar que no Brasil, a lista de doenças profissionais é constituída por uma relação agentes patogênicos ou de risco, a que estão expostos os trabalhadores em determinadas atividades, o que torna mais fácil a comprovação do nexo causal. Porém, a complexidade do problema ganha novos contornos ao adentrar no universo das doenças relacionadas ao trabalho, cujo nexo causal nem sempre está bem definido e acometem vários grupos de trabalhadores, como a hipertensão arterial e os DORT ${ }^{(11)}$.

Destacam-se no presente estudo, os Distúrbios Osteomusculares Relacionados ao Trabalho (DORT), cuja primeira referência oficial a esse grupo de afecções do sistema músculo-esquelético foi feita pela Previdência Social, com a terminologia tenossinovite do digitador, pela Portaria n. 4062, de 06/08/87. Em 1992 recebe a denominação Lesões por Esforços Repetitivos (LER), representando um dos grupos de doenças ocupacionais mais polêmicos no Brasil e em outros países. Nos últimos anos, têm sido, dentre as doenças ocupacionais registradas, as mais prevalentes, segundo estatísticas referentes à população trabalhadora segurada ${ }^{(12)}$.

Em princípio, uma doença que parecia se restringir a uma classe trabalhadora, estando relacionada apenas a execução de movimentos repetitivos, passou a invadir outros ramos profissionais, nos quais o trabalho não se caracterizava por repetição, merecendo destaque atualmente no cenário de adoecimento dos mais variados profissionais. 
A disseminação da doença implicou na mudança de denominação LER para Distúrbios Osteomusculares Relacionados ao Trabalho (DORT), ocorrida em 1997, proposta pelo Instituto Nacional de Seguridade Nacional (INSS), na revisão da Norma Técnica de avaliação para incapacidade de 1993, introduzindo novos elementos na análise da perícia médica do INSS acerca do processo de adoecimento ${ }^{(13)}$.

A nova terminologia utilizada (DORT) passou a incluir as tenossinovites, tendinites, sinovites, síndromes compressivas dos nervos periféricos, além de sintomatologias mais disseminadas, como a síndrome miofascial, fibromialgia e distrofia simpático-reflexa ${ }^{(14)}$.

Embora não sejam doenças recentes, as LER/DORT vêm, sem dúvida, assumindo um caráter epidêmico, sendo algumas de suas patologias crônicas e recidivas, de terapia difícil, gerando uma incapacidade para a vida que não se resume apenas ao ambiente de trabalho ${ }^{(15)}$.

Os fatores que favorecem a ocorrência dos DORT são múltiplos, constituindo um conjunto complexo, isolados ou agrupados, mas interligados, que exercem seu efeito simultaneamente na gênese da doença, tendo como sintomas, a dor localizada, irradiada ou generalizada, desconforto, fadiga e sensação de peso, formigamento, parestesia, sensação de diminuição de força, edema e enrijecimento articular. E, apesar de inicialmente apresentarem-se de forma insidiosa, predominando mais no término, em momentos de picos da produção e aliviarem com o repouso, com o decorrer do tempo podem tornar-se freqüentes durante o trabalho, inclusive incidindo nas atividades extra laborativas do trabalhador ${ }^{(14,16)}$.

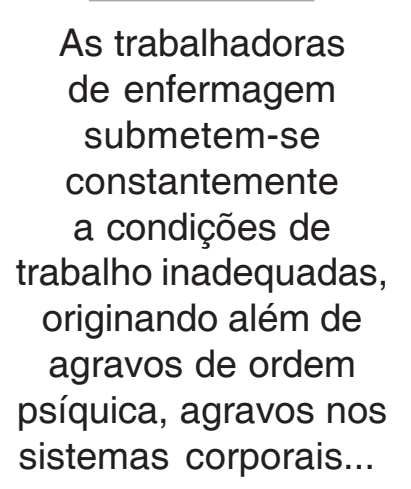

podem ser relacionados às lesões osteomusculares, tais como recursos tecnológicos inadequados, incluindo mobiliário, a falta de equipamentos especiais para movimentar pacientes, além da escassez de recursos humanos e a falta de treinamento ${ }^{(6)}$.

Somado às condições do ambiente hospitalar, ao analisar as atribuições que são impostas às trabalhadoras de enfermagem, pode-se observar características como polivalência de atividades, fragmentação, sobrecarga e aceleração do ritmo de trabalho, trazendo condições que nem sempre podem ser mensuráveis como doença ou acidentes, no entanto, prejudicam imensamente essas mulheres.

Assim, as trabalhadoras de enfermagem submetem-se constantemente a condições de trabalho inadequadas, originando além de agravos de ordem psíquica, agravos nos sistemas corporais, ocasionado os acidentes do trabalho e as licenças para tratamento de saúde ${ }^{(18)}$.

A maioria das queixas de saúde nos trabalhadores de enfermagem relacionam-se ao sistema osteomuscular, atribuídas principalmente a fatores ergonômicos e posturais inadequados, presentes na dinâmica hospitalar evidenciando a gravidade deste problema nesta categoria ${ }^{(6,19)}$.

Os estudos sobre as LER descrevem que os fatores de risco relacionados ao trabalho mais comumente citados como determinantes do aparecimento da doença, são os fatores biomecânicos (movimentos e posturas de risco que caracterizam a carga fisiológica podem estar presentes nos mais diferentes momentos da atividade laboral) e os psicossociais (pressão no trabalho, baixa autonomia, competitividade, entre outros) ${ }^{(20)}$.
A questão da gênese da LER-DORT continua sendo um desafio a ser superado devido aos conflitos e controvérsias que envolve pesquisadores, profissionais da saúde e trabalhadores ${ }^{(1)}$.

Mesmo com o diagnóstico de DORT e existindo uma legislação que obrigue o registro da Comunicação de Acidente do Trabalho (CAT), apenas $2 \%$ das empresas o fazem, dificultando a garantia dos direitos do trabalhador ${ }^{(17)}$.

Infelizmente, existe uma dificuldade para se comprovar o nexo causal da doença, ou seja, embora existam inúmeras condições desfavoráveis no trabalho que podem estar relacionadas ao aparecimento dos DORT nos trabalhadores, o registro oficial da doença nem sempre é obtido, gerando a subnotificação dos dados, impedindo que os números estatísticos correspondam à realidade.

\section{A TRABALHADORA DE ENFERMAGEM E OS DORT}

No ambiente hospitalar, existem vários fatores ergonômicos relacionados com problemas ambientais e organizacionais que
As condições citadas acima representam uma constante no trabalho dessas mulheres trabalhadoras, na realização de procedimentos relativos à assistência de enfermagem, como na higiene dos pacientes, na arrumação de leitos, realização de curativos, transporte e manipulação de pacientes, entre outras, assim como procedimentos relativos à gerência, como o preenchimento de intermináveis impressos, as anotações em prontuário, realização de escalas e gráficos de movimento de pacientes ou de cirurgia, todas atividades que, de uma forma ou de outra, podem contribuir para a gênese de um distúrbio osteomuscular.

Na verdade, a literatura é unânime ao descrever que não há uma causa única para a ocorrência de DORT, vários são os fatores, além da intensidade, duração e frequiência dos mesmos presentes no trabalho que podem concorrer para o seu surgimento, desconsiderando a diversidade de homens e mulheres ${ }^{(12)}$.

No entanto, sob as relações de gênero, o acometimento quantitativo maior da DORT expressa-se, sobretudo através da mulher trabalhadora, fato relacionado não somente às 
respostas biológicas, mas ao papel e à forma de inserção da mulher nas divisões social e sexual do trabalho ${ }^{(5,15)}$.

Freqüentemente observamos mulheres exercendo dupla e até tripla jornada, quando consideramos o trabalho doméstico relevante nos agravos à saúde das trabalhadoras.

Razão pela qual é necessário ir além da divisão do trabalho na busca de se compreender a desigualdade na distribuição dos DORT, para situá-las no campo das relações de gênero, redefinidas, por sua vez, pelas novas formas de organização do trabalho, nas quais, sem dúvida, as condições têm se revelado particularmente mais deletérias à saúde das mulheres ${ }^{(15)}$.

É importante que em pesquisas sobre gênero, trabalho e saúde, as situações de vida sejam avaliadas como um todo, contemplando as interações que se verificam entre trabalho doméstico e remunerado sendo este um aspectochave na compreensão do impacto diferenciado das condições de trabalho sobre a saúde de homens e mulheres, lembrando a agudização do peso da tripla carga de trabalho imposta às mulheres ${ }^{(5,21-22)}$.

Dada a relevância de se diagnosticar a situação, é necessário também que todas as questões acerca do ser mulher e trabalhar na enfermagem sejam consideradas, possibilitando intervenções reais que possam minimizar os riscos inerentes às trabalhadoras, haja vista a sobrecarga imposta não apenas pelo ambiente profissional como pelas atividades necessárias à organização do trabalho doméstico.

\section{REFERÊNCIAS}

1. Murofuse NT, Marziale MHP Doenças do sistema osteomuscular em trabalhadores de enfermagem. Rev Lat Am Enferm. 2005;13(3):364-73.

2. Marziale MHP. Enfermeiros apontam as inadequadas condições de trabalho como responsáveis pela deterioração da qualidade da assistência de enfermagem. Rev Lat Am Enferm. 2001;9(3):1-5.

3. Napoleão AA, Robazzi MLCC, Marziale MHP, Hayashida M. Causas de subnotificação de acidentes do trabalho entre trabalhadores de enfermagem. Rev Lat Am Enferm. 2000;8(3):119-20.

4. Reis JR, La Rocca PF, Silveira AM, Bonilla IML, Giné NA, Martín M. Fatores relacionados ao absenteísmo por doença em profissionais de enfermagem. Rev Saúde Pública. 2003;37(5):616-23.

5. Rocha LE, Debert-Ribeiro M. Trabalho, saúde e gênero: um estudo comparativo sobre analistas de sistemas. Rev Saúde Publica. 2001;35(6):539-47.

6. Parada EO, Alexandre NMC, Benatti MCC. Lesões ocupacionais afetando a coluna vertebral em trabalhadores de enfermagem. Rev Lat Am Enferm. 2002;10(1):64-9.

\section{CONSIDERAÇÕES FINAIS}

A gênese das LER/DORT apresenta relação com uma variedade de atividades, intensidade do ritmo de trabalho, além da própria forma de organização do trabalho. Nesse sentido, o trabalho exercido na enfermagem apresenta aspectos possivelmente relacionados à ocorrência dessas lesões, porém, um fator complicante é a comprovação desta relação, o que intensifica sofrimento dos acometidos além de gerar a subnotificação dos dados.

Não raramente, a equipe de enfermagem atua em instituições hospitalares que apresentam déficits de recursos humanos e materiais tornando a execução do trabalho mais penosa, gerando uma série de agravos a saúde.

As queixas de saúde relacionadas ao aparelho osteomuscular representam uma das maiores causas de sofrimento nos trabalhadores de enfermagem, sendo que estes valores assumem proporções maiores sobre as mulheres trabalhadoras, fator que justifica-se não somente pela fragilidade biológica inerente a mulher, mas em especial pela sua inserção social no mundo do trabalho.

À medida em que a mulher conquista novos papéis na sociedade, torna-se imprescindível considerar pois a questão do gênero na avaliação da saúde dos trabalhadores, para que nas investigações sobre o acometimento de trabalhadores de enfermagem por LER/DORT, sejam repensadas estratégias de intervenção no processo de adoecimento das mulheres trabalhadoras, já que elas são o grupo sob o qual mais incide a doença.

7. Drummond AS, Sampaio RO, Rodrigues GC. Distúrbios Osteomusculares Relacionados ao Trabalho (DORT): estudo com a equipe de enfermagem em uma unidade de terapia intensiva de pós-operatório (UTI-PO). Rev Socerj [periódico na Internet]. 2003 [citado 2005 mar. 16];16 Supl A:[cerca de 10 p.]. Disponivel em: http// www.socerj.org.br/temas/21enfer.pdf/

8. Queiroz MF, Maciel RH. Condições de trabalho e automação: o caso do soprador da indústria vidreira. Rev Saúde Pública. 2001;35(1):1-9.

9. Antunes R. Adeus ao trabalho? Ensaio sobre as metamorfoses e a centralidade do mundo São Paulo: Cortez; 2000.

10. Ribeiro HP. A violência oculta do trabalho: as lesões por esforços repetitivos. Rio de Janeiro: FIOCRUZ; 1999.

11. Dias EC. Aspectos atuais da saúde do trabalhador no Brasil. In: Rocha LE, Rigotto RM, Buschinelli JTP, organizadores. Isto é trabalho de gente? Vida, doença e trabalho no Brasil Petrópolis: Vozes; 1994. p. 139-56. 
12. Brasil. Ministério da Saúde. Secretaria de Políticas de Saúde. Protocolo de investigação, diagnóstico, tratamento e prevenção de Lesão por Esforços Repetitivos/Distúrbios Osteomusculares Relacionados ao Trabalho. Brasilia; 2000.

13. Verthein MAR, Minayo-Gomez C. A construção do sujeito-doente em LER. Hist Cienc Saude Manguinhos. 2000;7(2):329-47.

14. Brasil. Ministério da Saúde. Departamento de Ações e Estratégias. Área Técnica de Saúde do Trabalhador. Lesões por Esforços Repetitivos (LER) e Distúrbios Osteomusculares Relacionados ao Trabalho (DORT). Brasília; 2001.

15. Salim CA. Doenças do trabalho: exclusão, segregação e relações de gênero. São Paulo Perspect. 2003;17(1):11-24.

16. Oliveira RMR. A abordagem das Lesões por Esforços Repetitivos/Distúrbios Osteomusculares Relacionados ao Trabalho - LER/DORT no centro de referência em saúde do trabalhador do Espírito Santo [dissertação]. Rio de Janeiro: Escola Nacional de Saúde Pública, Fundação Oswaldo Cruz; 2001.

17. ONeill MJ. As mulheres e o risco da LER [texto na Internet]. [citado 2005 set. 26]. Disponível em: http://wwwprevler?artigos/mulheres
18. Barboza DB, Soler ZASG. Afastamentos do trabalho na enfermagem: ocorrências com trabalhadores de um hospital de ensino. Rev Lat Am Enferm. 2003;11(2):177-83.

19. Alexandre NMC, Moraes MA, Mahayri N. Modelo de um curso de orientação sobre determinados aspectos ergonômicos e posturais no trabalho do pessoal de enfermagem. Rev Bras Enferm. 1991;19(74):1-5.

20. Bernardino MTSM. Lesões por Esforços Repetitivos - LER: a doença para o indivíduo [dissertação]. São Paulo: Faculdade de Saúde Pública, Universidade de São Paulo; 1998.

21. Marcondes WB, Rotenberg L, Portela LF, Moreno CRC. O peso do trabalho leve feminino à saúde. São Paulo Perspec. 2003;17(2):91-101.

22. Fonseca RMGS. Equidade de gênero e saúde das mulheres. Rev Esc Enferm USP. 2005; 39(4):450-9. 